\title{
Endovascular and Operative Treatment of the Aortic Arch in a High-Risk Marfan Patient
}

\author{
Marwan Hamiko ${ }^{1}$ Matthias Endlich ${ }^{1}$ Wolfgang Schiller ${ }^{1} \quad$ Armin Welz $^{1}$ Georg Nickenig ${ }^{2} \quad$ Chris Probst $^{1}$ \\ ${ }^{1}$ Department of Cardiac Surgery, University of Bonn, Bonn, Germany \\ ${ }^{2}$ Department of Cardiology, University of Bonn, Bonn, Germany \\ Address for correspondence Marwan Hamiko, MD, Department of \\ Cardiac Surgery, University of Bonn, Bonn, Germany \\ (e-mail: marwan.hamiko@ukb.uni-bonn.de).
}

Thorac Cardiovasc Surg Rep 2016;5:68-70.
Abstract
Keywords
- aortic disease
- aneurysm
- aorta/aortic
- endovascular procedures/stents
- stents

In this case, we describe a combined endovascular and operative management for aortic arch repair in a 57-year-old Marfan patient with complex aortic arch geometry previously treated with several open surgeries for acute type A dissection. The patient, who was presented to our department with dorsal pain, deemed to be at high operative risk for another open aortic surgery due to massive aortic calcification. It is an unusual method of placing a custom-made stent-graft system in the false aortic lumen with operative and endovascular treatment of the supra-aortic vessels.

\section{Introduction}

Marfan syndrome, an autosomal dominant disorder, is a disease of the connective tissue, especially with manifestation of the cardiovascular system. Aneurismal formation, dissection, and rupture of the aorta may be consequences of the disease with a high mortality. Despite early correction, the disease is not curative but progressive. Several redo surgeries or interventions with high mortality are common in Marfan patients. Endovascular therapy is controversial discussed as an alternative option for Marfan patients. Some case reports and series describe the experience of endovascular procedures in Marfan patients. ${ }^{1,2}$ However, in cases of involved aortic arch, endovascular repair could be difficult because it needs surgical debranching of the supraaortic trunk vessels. In these cases, custom-made stent-graft systems could help treating complex aortic geometries.

We describe a combined endovascular and operative management for aortic arch repair in a Marfan patient with complex aortic arch geometry previously treated with several open surgeries for acute type A dissection and who deemed to be at high operative risk for another open aortic surgery due to massive aortic calcification. It is an unusual method of placing a custom-made stent-graft system in the false aortic lumen $(\mathrm{FL})$ with operative and endovascular treatment of the supra-aortic vessels.

received

December 1, 2015

accepted after revision

December 14, 2015

published online

January 29, 2016

\section{Case Presentation}

A 57-year-old female Marfan patient with a medical history of treatment for acute type A dissection with a mechanical Bentall procedure and coronary artery bypass in 1991 was admitted to our department with dorsal pain. In 1998 and 2001, redo surgeries were necessary due to pseudoaneurysm formation including a covered rupture at the distal Bentall anastomosis. In 2009, the patient had also undergone another surgery, after heavily calcification of the residual intimal flap with a pressure gradient of $100 \mathrm{~mm} \mathrm{Hg}$ in the aortic arch.

The computer tomography (CT) scan shows a dilatation of the aortic arch up to $8.3 \mathrm{~cm}$ with signs of a new covered rupture ( - Fig. 1A) and complex dissection of the supra-aortic vessels with a porcelain aorta (-Figs. 1B and 2A, B). A preoperative angiogram showed multiple reentries between the true lumen (TL) and FL. An occlusion of the TL was impossible. Therefore, we planned to create a stent-graft system for placing in the FL. Due to the patient's high-risk profile, conventional surgery was refused and a minimally invasive hybrid-endovascular and operative approach was planned.

A three-dimensional (3D) reconstruction of the $\mathrm{CT}$ scan was required to create a custom-made stent-graft system. A silicon 3D model, exactly corresponding to the patient's aorta was manufactured ( - Fig. 3 ), and the fenestrated tapered stent-graft

\footnotetext{
(c) 2016 Georg Thieme Verlag KG Stuttgart · New York
}

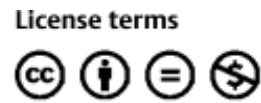



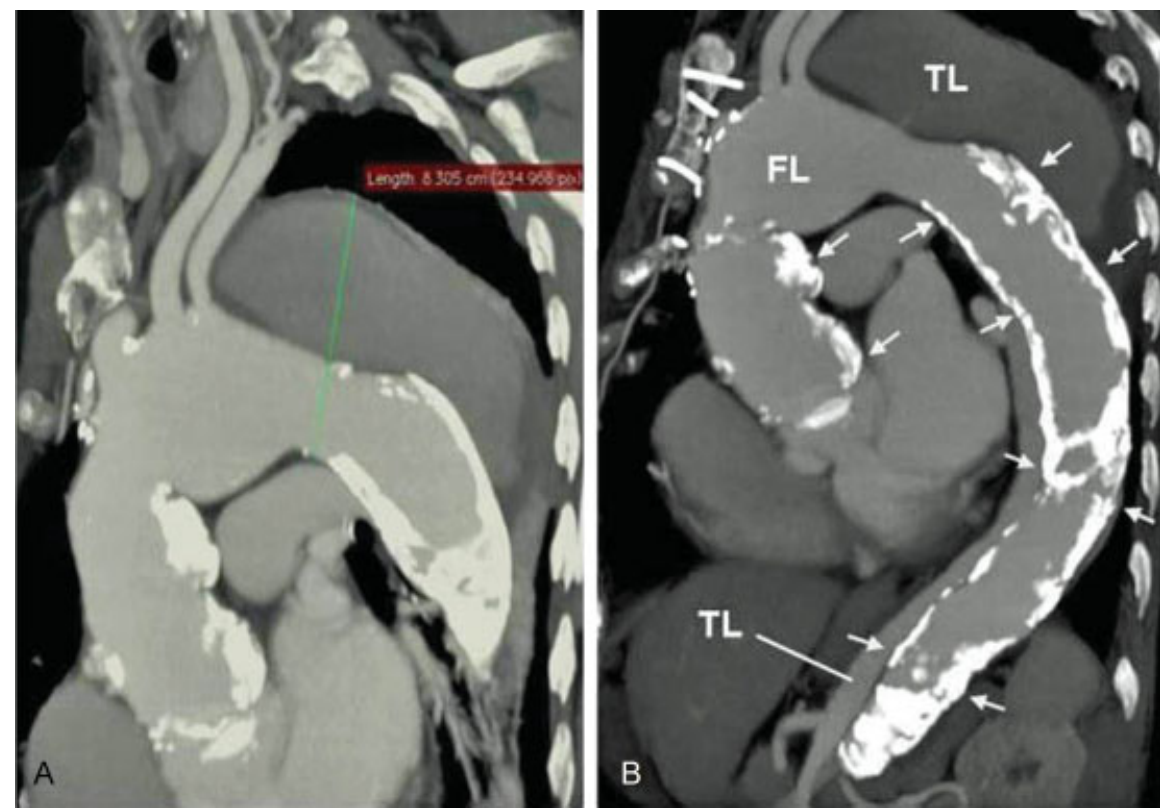

Fig. 1 (A) Preoperative CT scan of the aneurysmal dilatation of the aortic arch up to $8.3 \mathrm{~cm}$. The image shows also the origin of the supra-aortic vessels. (B) The image shows the heavily calcification of the aorta (white arrows). CT, computed tomography; FL, false lumen; TL, true lumen.
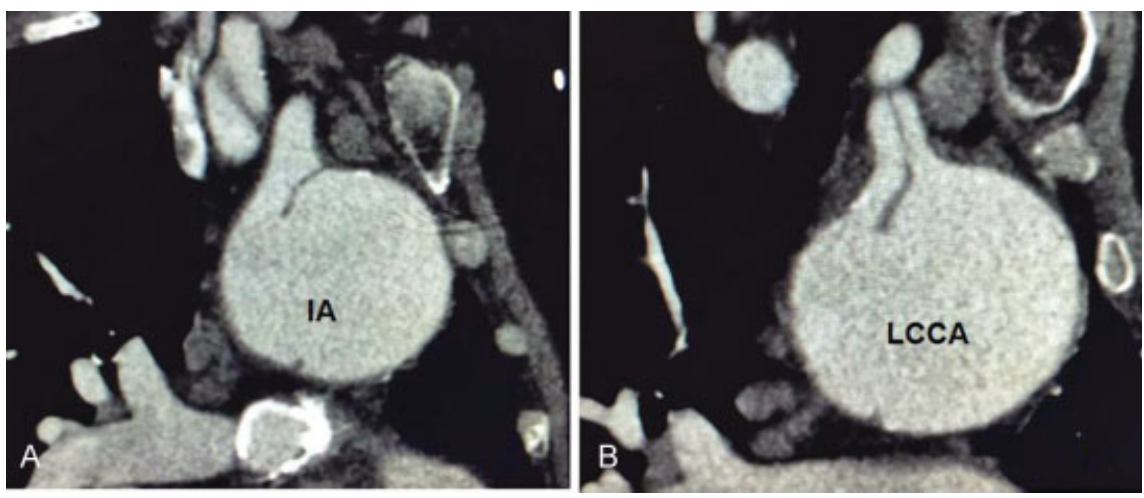

Fig. 2 Cross section of the aortic arch at the level of the innominate artery (IA; A) and for the left common carotid artery (LCCA; B). Both images show the dissected branches.

system (length: $260 \mathrm{~mm}$; proximal diameter: $46 \mathrm{~mm}$; distal diameter: $34 \mathrm{~mm}$; Bolton Medical Inc., Florida, United States) was designed. The graft was constructed with two side holes corresponding to the innominate artery (IA) and the left common carotid artery (LCCA).

First stage of the procedure involved creation of bilateral subclavian-carotid bypasses. After heparinization, an activating clotting time (ACT) of $>200$ seconds and partial clamping, an $8.0 \mathrm{~mm}$ synthetic graft (Hemashield, Maquet Getinge Group, Rastatt, Germany) was anastomosed in an end-toside fashion from the carotid to the subclavian artery.

A 6-Fr sheath was then placed into the right femoral artery. Through this sheath, a soft wire (Terumo, Terumo Corp., Tokyo, Japan) was positioned in the FL reaching the ascending aorta and was exchanged over a pigtail catheter by a double curved extrastiff Lunderquist wire (Cook Inc., Bloomington, Indiana, United States). Similarly, another 6-Fr sheath was placed into the left femoral artery and another pigtail catheter was established in the TL of the descending aorta.
Through another sheath, placed in the right subclaviancarotid bypass, a third pigtail catheter marked the origin of the IA. Over the stiff Lunderquist wire, the custom-made graft system was advanced through the FL with its proximal part in the TL of the ascending aorta, $5 \mathrm{~cm}$ above the aortic valve and its distal part ending in the FL. After confirming the side holes in the correct position, the graft was deployed under rapid pacing and hypotension condition.

Then, two Gore ViaBahn grafts (Gore, Flagstaff, Arizona, United States) with 10.0 and $7.5 \mathrm{~cm}$ length, respectively, were positioned via the side holes into the right carotid artery and LCCA. After these stages, a 22.0-mm AMPLATZER Vascular Plug (AGA Medical, Golden Valley, Minnesota, United States) occluded the proximal part of the TL of the dissected descending aorta. Perfusion of the abdominal organs was carried retrograde through several re-entries and a large distal entry tear at the level of the renal arteries. The right and left subclavian arteries were then occluded at their origin by two additional AMPLATZER Vascular Plugs. 


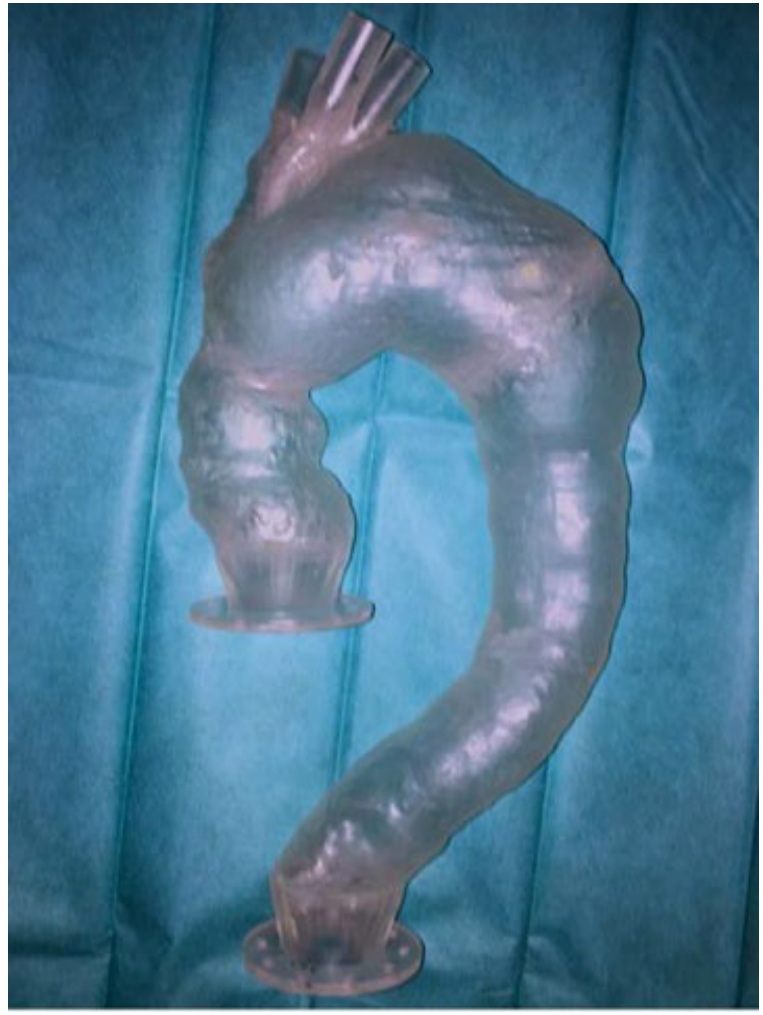

Fig. 3 A silicon three-dimensional model with exact correspondence to the patient's aorta.

The postoperative course was uneventful. The patient did not experience any symptoms of upper-extremity ischemia. A CT scan before discharge showed satisfactory results with a complete exclusion of the large aneurysm and sufficient supply of the supra-aortic vessels (-Fig. 4).

\section{Discussion}

Surgical treatment of the aortic arch is a challenging procedure, especially in patients with a complex previous surgeries and aortic anatomy. A lot of methods have been developed during the last years to treat aortic arch pathologies. Hybrid aortic arch procedures, endovascular aortic arch repair with fenestrated stent grafts, or in situ fenestration have all been introduced as alternative options for managing complex aortic arch pathologies. ${ }^{1-3}$

Endovascular treatment has become more popular also in Marfan patients but is still in controversial discussion. Early experiences of endovascular management of aortic arch pathologies in Marfan patients showed beneficial results. ${ }^{1,2}$

Recently, Botta et al presented their experience of reconstructing the aortic arch treating a penetrating atherosclerotic ulcer in a 75-year-old patient. ${ }^{4}$ They also used a custommade branched thoracic stent-graft system manufactured by Bolton Medical. First experience with multibranched stentgraft systems for repairing aortic arch aneurysms were described by Lioupis et al. ${ }^{5}$ They successfully demonstrated the technical feasibility of branched stent-graft systems to treat aortic arch aneurysms in six patients.

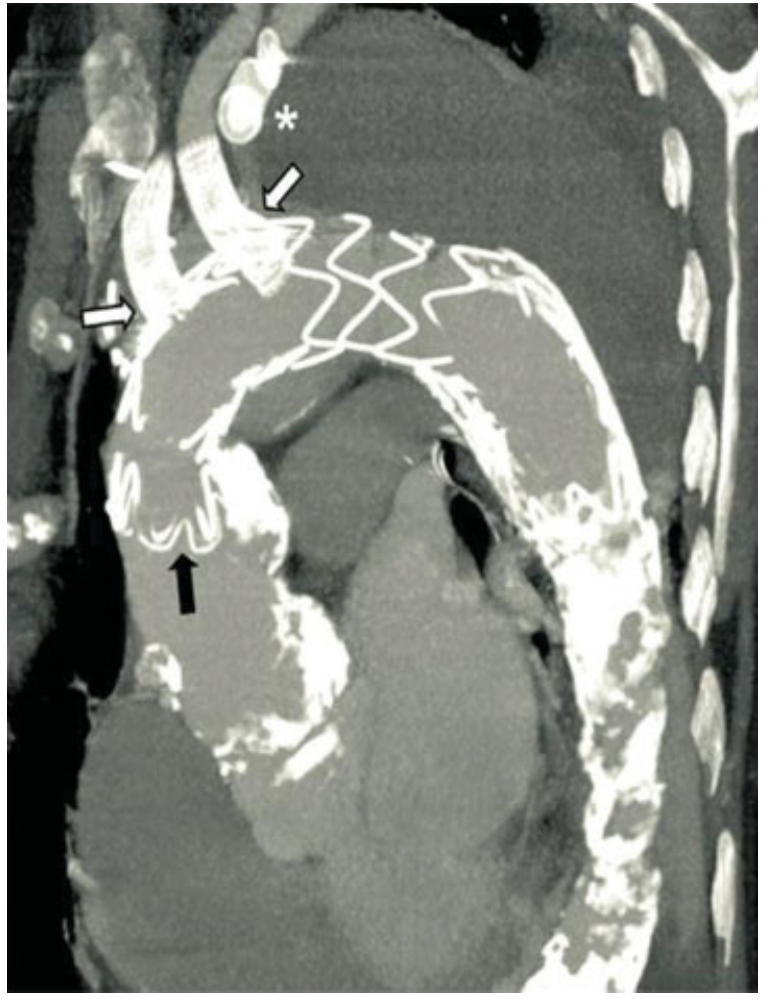

Fig. 4 Postoperative CT scan shows a successful reconstruction of the aortic arch with a new custom-made stent-graft system placed in the false lumen with occlusion of the true lumen, left and right subclavian arteries. The dark arrow marks the stent graft with its origin in distal aorta ascendens. The white arrows mark the two Gore ViaBahn grafts positioned in the right and left common carotid arteries. The supra-aortic vessels were sufficiently supplied through the implanted grafts. * marks the occlusion of the left subclavian artery with an AMPLATZER Vascular Plug. CT, computed tomography.

According to the high-risk profile of our patient, we decided to treat the covered rupture with this complex hybrid procedure by positioning a custom-made stent graft in the FL and occluding the TL of a dissected aorta. Due to the large reentries, an occlusion of the TL was impossible. In the literature, such a case has not been described yet.

\section{References}

1 Waterman AL, Feezor RJ, Lee WA, et al. Endovascular treatment of acute and chronic aortic pathology in patients with Marfan syndrome. J Vasc Surg 2012;55(5):1234-1240, 1240-1241

2 Akin I, Kische S, Rehders TC, et al. Current role of endovascular therapy in Marfan patients with previous aortic surgery. Vasc Health Risk Manag 2008;4(1):59-66

3 Inoue K, Hosokawa H, Iwase T, et al. Aortic arch reconstruction by transluminally placed endovascular branched stent graft. Circulation 1999;100(19, Suppl):II316-II321

4 Botta L, Fratto P, Cannata A, Bruschi G, Rampoldi A, Martinelli L. Aortic-arch Reconstruction with Bolton Medical Branched Thoracic Stent Graft. Euro J Vasc Endovasc Surg Extra 2013;25(5): e38-e41

5 Lioupis C, Corriveau MM, MacKenzie KS, Obrand DI, Steinmetz OK, Abraham CZ. Treatment of aortic arch aneurysms with a modular transfemoral multibranched stent graft: initial experience. Eur J Vasc Endovasc Surg 2012;43(5):525-532 